\title{
Design of the ILC Crab Cavity System
}

\author{
C. Adolphsen ${ }^{1}$, C. Beard ${ }^{2}$, L. Bellantoni ${ }^{3}$, G. Burt ${ }^{4}$, R. Carter $^{4}$, B. Chase ${ }^{3}$, M. Church ${ }^{3}$, A. Dexter ${ }^{4}$, M. \\ Dykes $^{2}$, H. Edwards ${ }^{3}$, P. Goudket ${ }^{2}$, R. Jenkins ${ }^{4}$, R. M. Jones ${ }^{5}$, A. Kalinin ${ }^{2}$, T. Khabiboulline ${ }^{3}, K_{\text {. Ko }}{ }^{1}$, A. \\ Latina $^{6}$, Z. Li $^{1}$, L. Ma ${ }^{2}$, P. McIntosh ${ }^{2}$, C. $\mathrm{Ng}^{1}$, A. Seryi ${ }^{1}$, D. Schulte ${ }^{6}$, N. Solyak ${ }^{3}$ I. Tahir ${ }^{4}$, L. Xiao $^{1}$ \\ 1. SLAC, Menlo Park, California \\ 2. Daresbury Laboratory, Warrington, UK, WA4 $4 A D$ \\ 3. FNAL, Batavia, Illinois, 60501 \\ 4. Cockcroft Institute, Lancaster University, Warrington, UK, WA4 4AD \\ 5. Cockcroft Institute, The University of Manchester, Warrington, UK, WA4 4AD \\ 6. CERN, Geneva, Switzerland
}

\begin{abstract}
The International Linear Collider (ILC) has a 14 mrad crossing angle in order to aid extraction of spent bunches. As a result of the bunch shape at the interaction point, this crossing angle at the collision causes a large luminosity loss which can be recovered by rotating the bunches prior to collision using a crab cavity. The ILC baseline crab cavity is a 9-cell superconducting dipole cavity operating at a frequency of $3.9 \mathrm{GHz}$. In this paper the design of the ILC crab cavity and its phase control system, as selected for the RDR ${ }^{1}$ in February 2007 is described in fuller detail.
\end{abstract}

\section{Introduction}

Because of the bunch-length to beam-width ratio envisioned for ILC bunches, the delivered luminosity at non-zero crossing angles depends critically on having precise "crabbing," i.e. rotation of the bunches around the vertical axis. The crab cavity design must provide a large transverse impedance at a frequency that is a multiple of the $1.3 \mathrm{GHz}$ main linac frequency, within a limited transverse dimension because of the proximity of the 'return' arms, i.e. the $e^{+}$arm for the cavity that crabs the $e^{-}$bunches. Superconducting RF technology has been adopted for the ILC crab cavity to enable more effective handling of long bunch trains in comparison to a more conventional normal conducting cavity solution. The peak magnetic fields on the inner surface of the cavity will be constrained by the ability of the niobium material to sustain the corresponding magnetic fields, and the aperture must not be so tight as to introduce wakefield effects. The system must also maintain a very precise control of the phasing of the 'crabbing' stations on the $e^{+}$and $e^{-}$arms of the collider. The transverse space constraints favour using as high a frequency as possible; experience at Fermilab has shown that SRF cavities at $3.9 \mathrm{GHz}$ can be successfully fabricated and run at full RF field. Accordingly, the ILC crab cavity design of the RDR is based on the $3.9 \mathrm{GHz}$ deflecting mode cavity initially developed at Fermilab for use in a $K^{+}$ separated beamline ${ }^{2}$. That design is a 13-cell cavity; here a 9-cell version is being developed. Two 9-cell cavities per arm, operated at $5 \mathrm{MV} / \mathrm{m}$ peak deflection, will provide enough rotation for a $500 \mathrm{GeV}$ beam with no redundancy or for a $250 \mathrm{GeV}$ 
beam with $100 \%$ redundancy. Table 1 lists some of the basic specifications of the superconducting crab cavity system.

Table 1: ILC Crab System Parameters.

\begin{tabular}{|c|c|}
\hline Crossing angle, mrad & 14 \\
\hline Number of cryovessels per IP & 2 \\
\hline Number of 9-cell cavities per cryovessel & 2 \\
\hline Active length of cavity, $\mathrm{m}$ & 0.344 \\
\hline Required bunch rotation , mrad & 7 \\
\hline Location of crab cavities from the corresponding IP, $\mathrm{m}$ & $13.4-17.4$ \\
\hline Longitudinal space allocated per cryovessel, $\mathrm{m}^{\mathrm{a}}$ & 3.8 \\
\hline $\mathrm{X}$ offset at IP due to crab cavity angle (R12), $\mathrm{m} / \mathrm{rad}$ & 16.3 \\
\hline Y offset at IP due to crab cavity angle (R12), m/rad & 2.4 \\
\hline Betatron X function at crab cavity, $\mathrm{km}$ & 12.7 \\
\hline Betatron Y function at crab cavity, km & 14.3 \\
\hline Betatron $X$ size at the crab cavity, $\mu \mathrm{m}$ & 510 \\
\hline Betatron Y size at the crab cavity, $\mu \mathrm{m}$ & 34 \\
\hline Amplitude at 1TeV CM, MV ${ }^{\mathrm{b}}$ & 2.64 \\
\hline Max amplitude with operational margin ${ }^{\mathrm{C}} \mathrm{MV}$ & 4.1 \\
\hline Cavity operating temperature, $\mathrm{K}$ & 1.8 \\
\hline
\end{tabular}

\section{Cavity Cell Shape}

The dimensions and profile of the cell shape are given in Figure 1 and Table 2. The end-cell dimensions are slightly different from the mid-cell dimensions to ensure field flatness. The field shapes, shown in Figures 2 and 3, are similar to those of a pillbox $\mathrm{TM}_{110}$ cavity; the cell-to-cell phase advance is $\pi$. The peak surface electric field, in comparison to the peak surface magnetic field, (shown along with other basic design parameters in Table 3) is much smaller for this field shape than it is for the field shapes typical of monopole cavities.

The surface current is peaked very sharply in the iris region, as shown in Figure 4. Azimuthal asymmetry is induced by putting a $1.5 \mathrm{~mm}$ deep (in radius) flat on the side of the cavity; this introduces a split of $\sim 10 \mathrm{MHz}$ between each polarisation of the dominant dipole modes.

The complicated field-flatness tuning technique of reference ${ }^{3}$ is not needed with a 9 cell cavity, as the $8 \pi / 9$ mode is $2.2 \mathrm{MHz}$ away from the operating mode.

\footnotetext{
${ }^{\mathrm{a}}$ The design presented here will need $\sim 2.3 \mathrm{~m} ; 3.8 \mathrm{~m}$ is required for a worst-case scenario of 63 -cell cavities per station.

${ }^{\mathrm{b}}$ The required deflection voltage is $V=c E \theta_{\mathrm{c}} /\left(4 \pi f R_{12}\right)$.

${ }^{\mathrm{c}}$ The maximum reasonably expected performance of 2 cavities. This is is higher than nominally required, to account for possible change of optics, system redundancy, and operational flexibility.
} 
Table 2: Cell Shape Parameters.

\begin{tabular}{|l|c|c|c|c|}
\hline & & mid-cell & trans-cup & end-cup \\
\hline half cell length & $\mathrm{g} / 2\left(\mathrm{Z}_{\mathrm{ir}}\right)$ & $19.2 \mathrm{~mm}$ & $19.2 \mathrm{~mm}$ & $18.6 \mathrm{~mm}$ \\
\hline iris radius & $\mathrm{a}\left(\mathrm{a}_{\mathrm{ir}}\right)$ & $15.0 \mathrm{~mm}$ & $15.0 \mathrm{~mm}$ & $18.0 \mathrm{~mm}$ \\
iris curvature & $\mathrm{r}_{\mathrm{i}}$ & $5.5 \mathrm{~mm}$ & $5.5 \mathrm{~mm}$ & $5.5 \mathrm{~mm}$ \\
\hline equator radius & $\mathrm{b}\left(\mathrm{a}_{\mathrm{eq}}\right)$ & $47.18 \mathrm{~mm}$ & $47.37 \mathrm{~mm}$ & $47.37 \mathrm{~mm}$ \\
equator curvature & $\mathrm{r}_{\mathrm{e}}$ & $11.41 \mathrm{~mm}$ & $11.41 \mathrm{~mm}$ & $11.41 \mathrm{~mm}$ \\
\hline
\end{tabular}

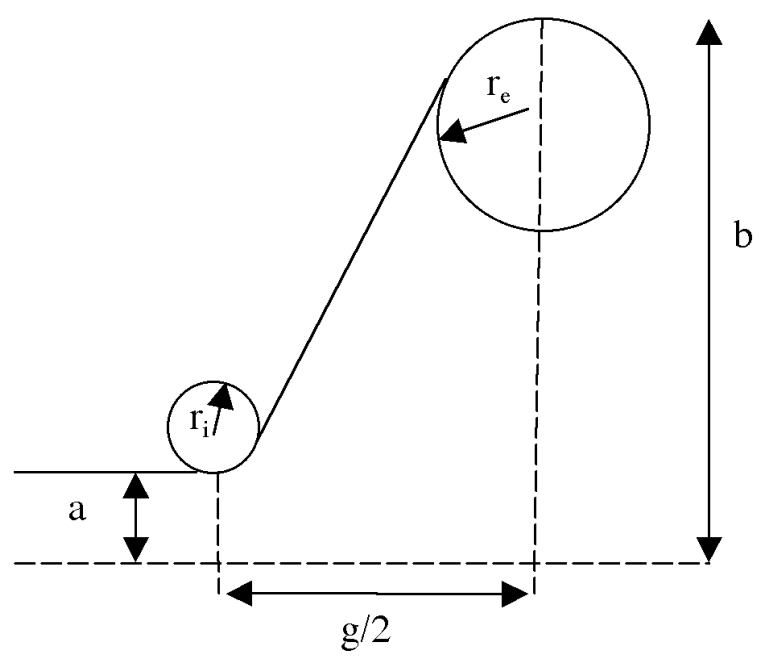

Figure 1: Cell Shape Parameters 


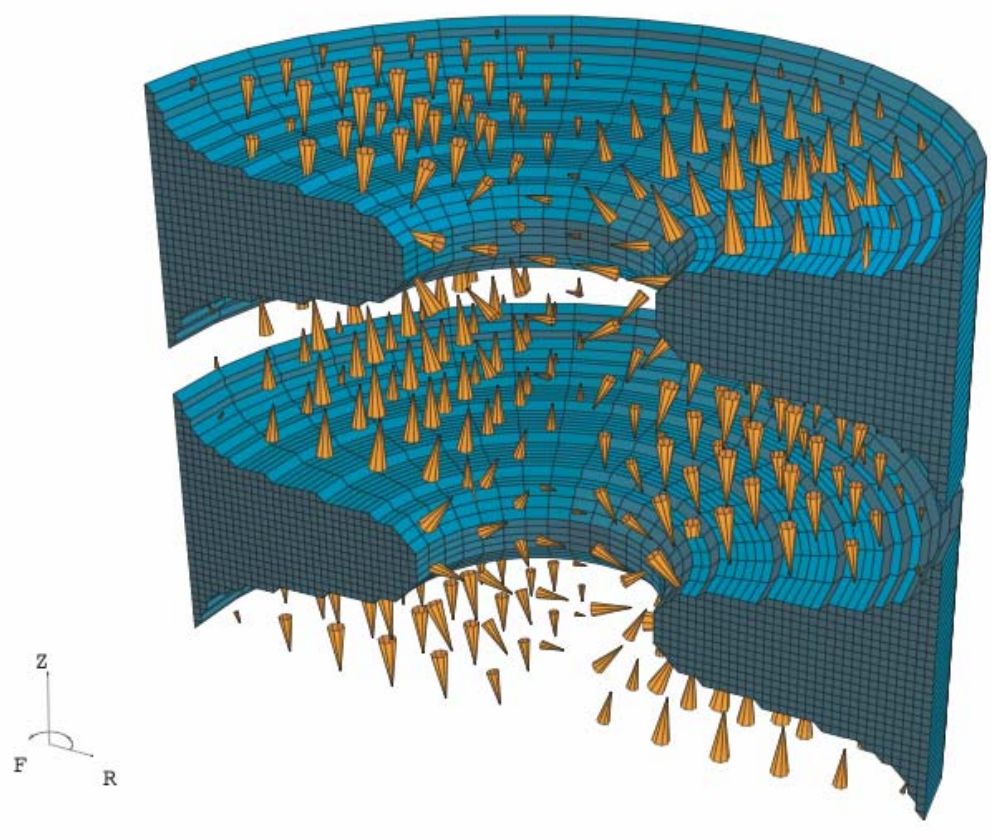

Figure 2: Electric Field

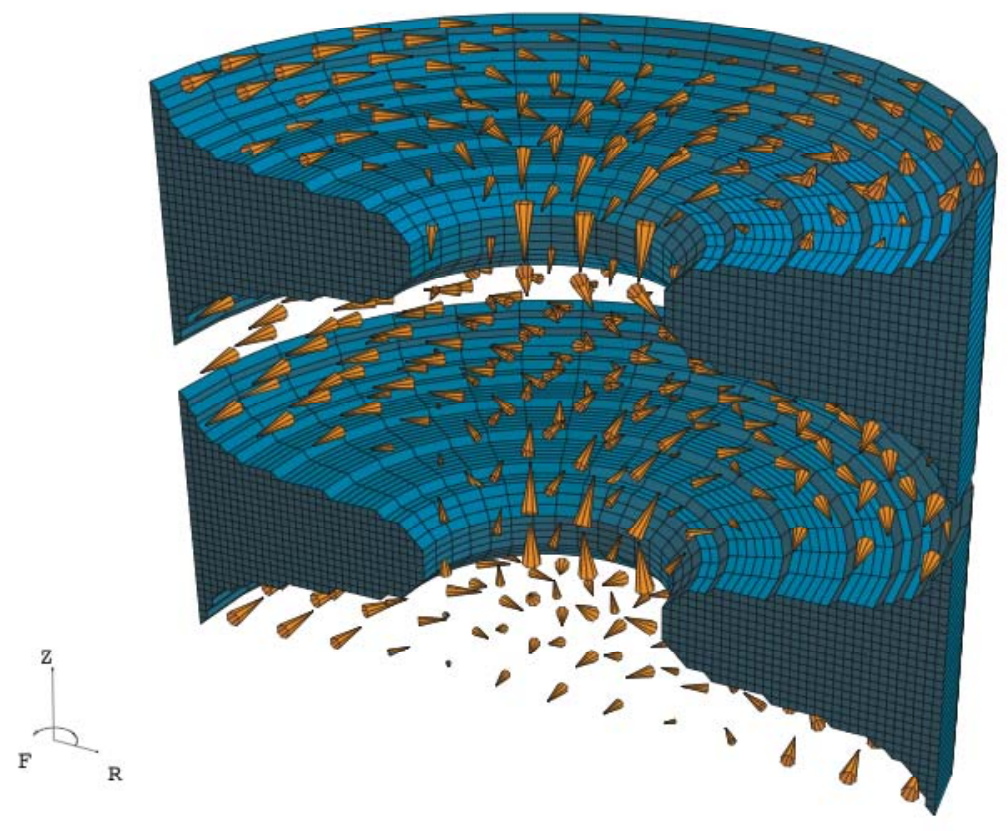

Figure 3: Magnetic Field Lines. 


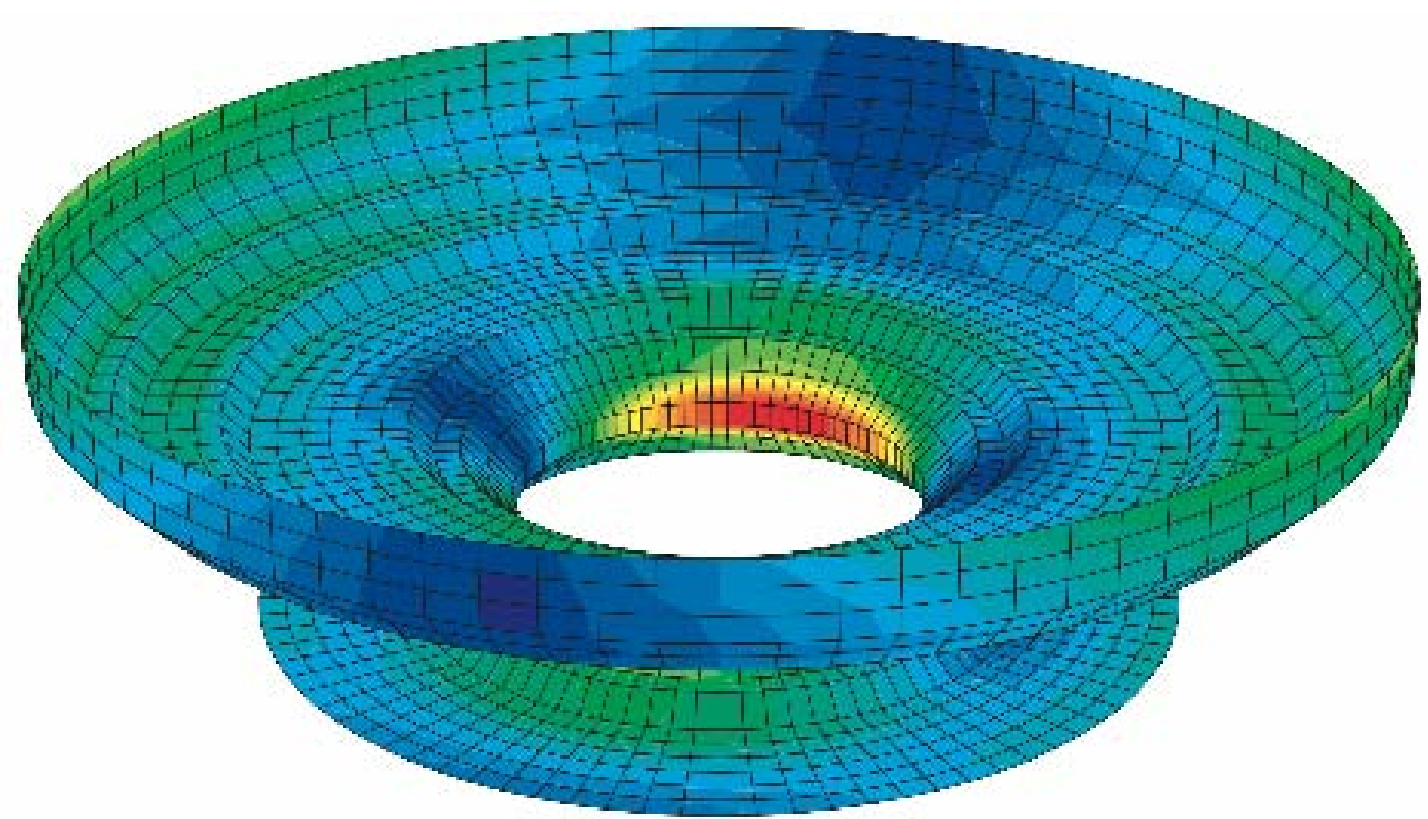

Figure 4: Surface Current Density.

\section{Prototype Performance}

The limiting factor to operating deflection gradient is expected to be the peak surface magnetic field around the iris which is $120 \mathrm{mT}$ at $7.5 \mathrm{MV} / \mathrm{m}$. That field level is typical of the levels achieved at many laboratories with small grain, Buffered Chemical Polished (BCP) treated niobium sheet material; the higher levels that have been reported with EP treated sheet are not really needed for this application. The forming of large grain sheet for these smaller cavities has not been investigated; the basic manufacturing technique of e-beam welding highly pure sheet niobium produced by industry is similar to and derived from the technology utilised in building 1.3 GHz cavities.

Figures 5 and 6 show the results of a cold test in January 2006 of a three cell prototype (serial number C15a-3C-4C; nickname "John”) manufactured by Advanced Energy Systems of Medford NY, U.S.A. This cavity was made from $2.2 \mathrm{~mm}$ thick eddy-current scanned RRR 300 niobium. Figure 5 shows the cavity surface $Q$ factor as a function of peak magnetic field in the surface. The $Q$ factor is determined from the ringdown time when RF power is removed; the magnetic field is inferred from the MAFIA finite element values of Table 3 and measurement of the energy flow into and out of the cavity at unity external coupling. Surface resistance, $\mathrm{R}_{\mathrm{s}}$, is inferred from $Q$ with the MAFIA value of $G=Q R_{s}$. 


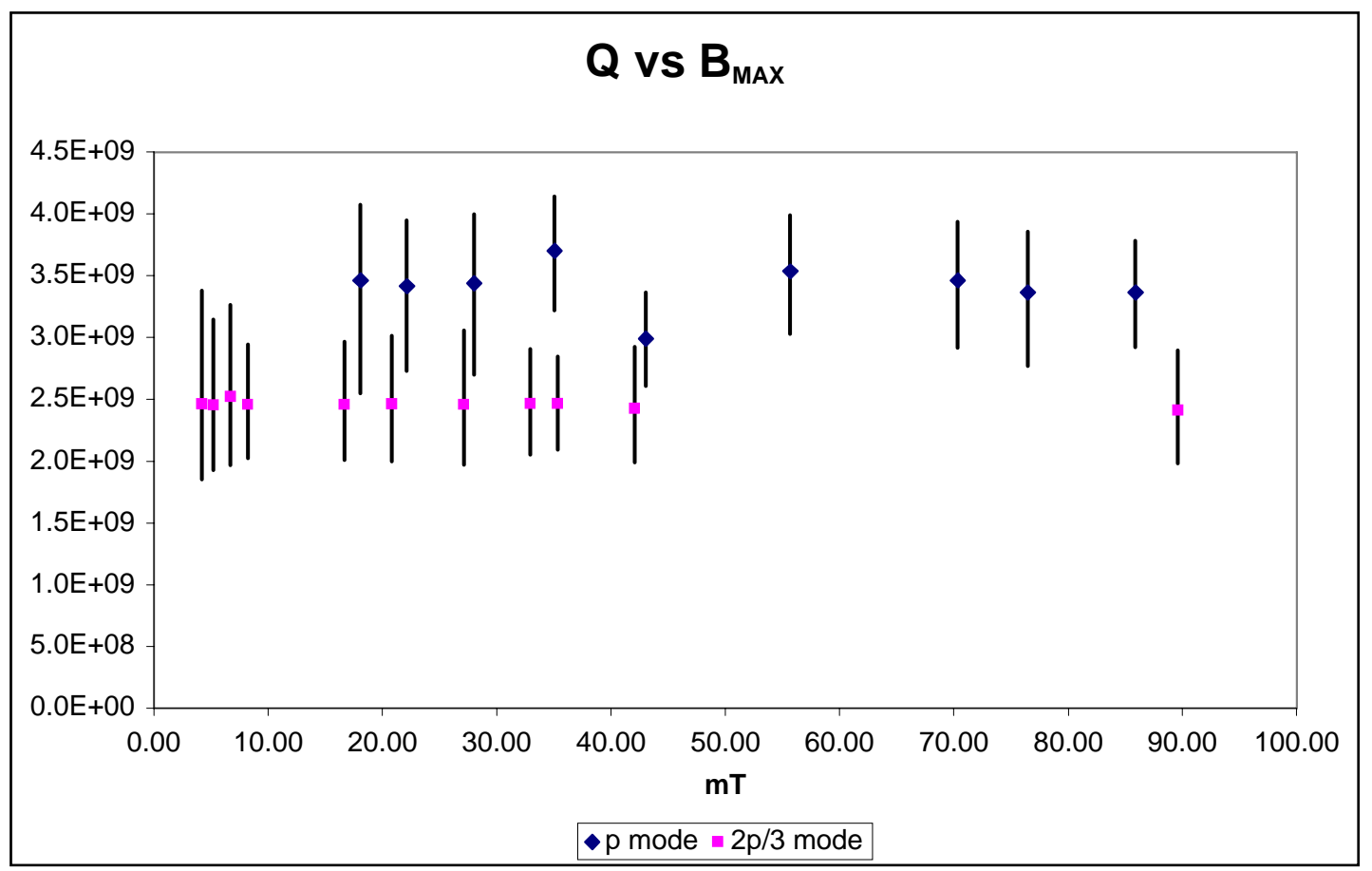

Figure 5: $Q$ vs. $B_{\text {MAX }}$ for “John”.

Table 3: Crab Cavity RF Parameters.

\begin{tabular}{|c|c|}
\hline At $5 \mathrm{MV} / \mathrm{m} \mathbf{P}_{\perp}$ : & \\
\hline$B_{\mathrm{MAX}}$ & $73 \mathrm{mT}$ \\
\hline $\begin{array}{c}E_{\mathrm{MAX}} \\
U\end{array}$ & $\begin{array}{c}16.6 \mathrm{MV} / \mathrm{m} \\
0.25 \mathrm{~J}\end{array}$ \\
\hline $\begin{array}{c}Q(\mathrm{Nb}, \text { room temp) } \\
(\mathrm{R} / \mathrm{Q})^{\prime}=\frac{1}{2} \frac{\left|V_{L}(r)\right|^{2}}{\omega U}\left(\frac{c}{\omega r}\right)^{2}\end{array}$ & 4780 \\
\hline$G=Q \times R_{\mathrm{SURF}}$ & $225 \Omega$ \\
\hline$R_{\mathrm{BCS}}$ (best measurement) @ 1.8K & $30 \mathrm{n} \Omega$ \\
\hline$R_{0}$ (best measurement) & $40 \mathrm{n} \Omega$ \\
\hline$Q @ 70 \mathrm{n} \Omega 1.8 \mathrm{~K}$ & $3.2 \times 10^{9}$ \\
\hline Surface power @ 70n $\Omega$ & $1.9 \mathrm{~W}$ \\
\hline
\end{tabular}


Figure 5 shows a relatively flat behaviour up to a quench point at about $90 \mathrm{mT}$ corresponding to peak field. This test was performed after the cavity received a $80 \mu \mathrm{m}$ manufacture-phase BCP etch followed by a single $15 \mu \mathrm{m}$ light etch. A test of "Thick" in August of 2004 went up to a peak field of $120 \mathrm{mT}$. The flat behaviour is not well understood in that (in principle) the performance of this cavity should be limited by heating of the inner wall; in that case the curve should drop off quadratically. However, this simple thermal model fails to explain the $Q$ vs $E$ curves of other cavity designs as well.

Figure 6 shows the temperature dependence of the surface resistance. The qualitative BCS dependence is clear and the low-temperature limiting surface resistance appears on the right of the plot. The BCS curve is consistent with data taken at DESY during the development of the $1.3 \mathrm{GHz}$ cavity. It is also consistent with data taken in the same Dewar during the development of the $3.9 \mathrm{GHz}$ " $3^{\text {rd }}$ harmonic" cavity under development at FNAL for use at DESY in the FLASH and XFEL facilities. The value $R_{0}=39 \pm 6 \mathrm{n} \Omega$ is not unreasonable, although somewhat larger than similar FNAL taken data from the $3^{\text {rd }}$ harmonic cavity fabrication. The residual magnetic field inside the Dewar is about $25 \mathrm{mG}$, and one would expect, as a rule of thumb, an $R_{0}$ contribution of about $20 \mathrm{n} \Omega$ from this effect. That prediction is very approximate however, and there could be other contributions to $R_{0}$.

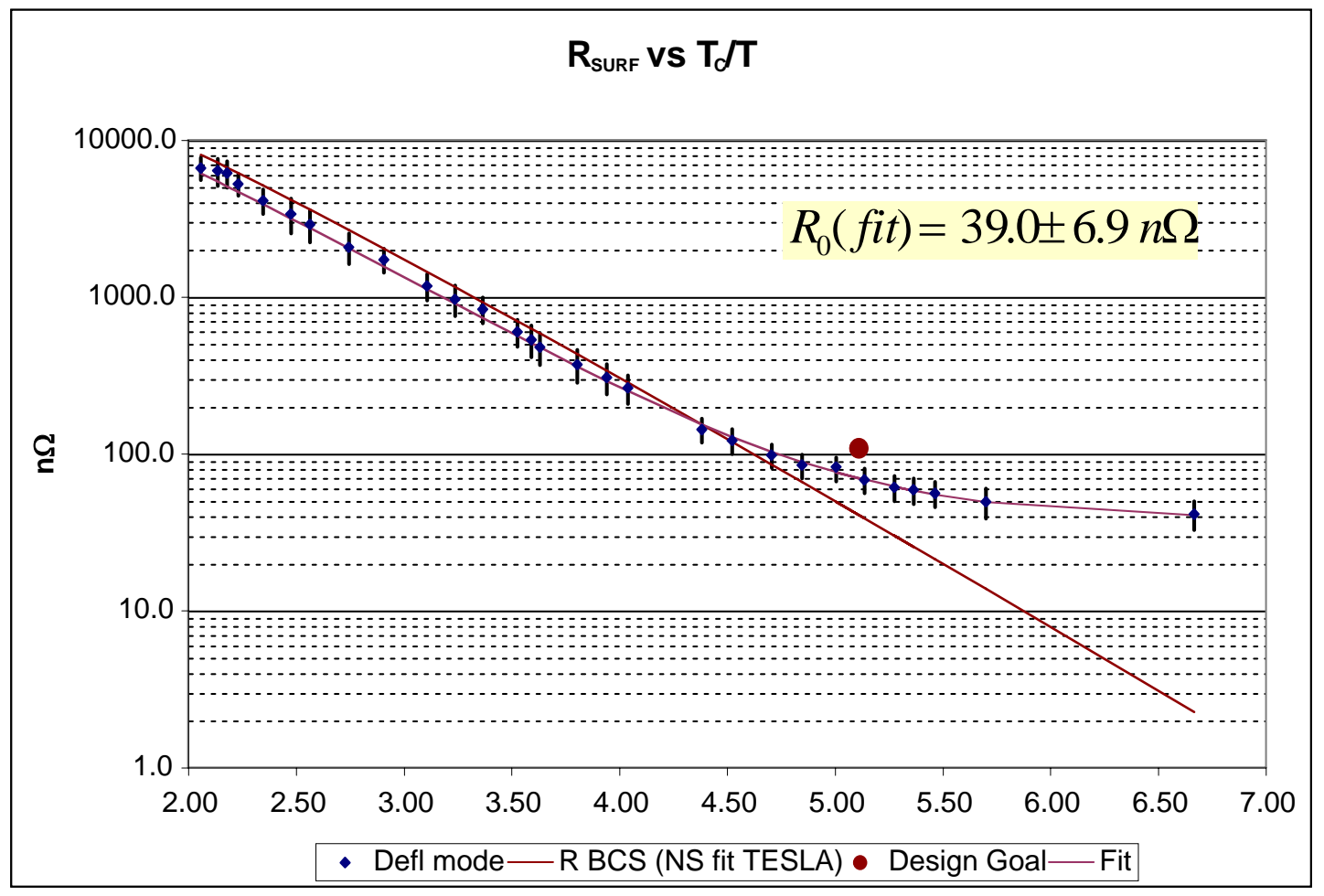

Figure 6: $R_{\mathrm{SURF}}$ vs. $T_{\mathrm{C}} / T$ for “John”. 


\section{Mode Structure and Wakefields}

The beampipe has $36 \mathrm{~mm}$ diameter, and below the cut-off frequencies of 6380 $\mathrm{MHz}$ for monopole modes and $4880 \mathrm{MHz}$ for dipole modes, simple analytical models can be used for the dispersion curves.

The $\mathrm{TM}_{010}$ modes fit:

$$
f=f_{0} \sqrt{1+2 k(1-\cos \phi)}
$$

with $f_{0}=2784 \mathrm{MHz}$ and $k=9.03 \times 10^{-3}$, with a $\pi$ mode at the high end of the passband. The modes with the highest coupling to the beam are the ones nearest the intersection of the dispersion with the light cone; this happens at $130^{\circ}$ phase advance, $2826 \mathrm{MHz}$. For the $\mathrm{TM}_{020}$ band, these numbers are $5918 \mathrm{MHz}, 22.14 \times 10^{-3}, 82^{\circ}$, and $6029 \mathrm{MHz}$, with a 0 mode at the low end of the passband. The $\mathrm{TM}_{020}$ dispersion curve is not quite as close to the fitted function as that of the $\mathrm{TM}_{010}$ mode; for $\mathrm{TM}_{020}$, the residual is typically 7-8 $\mathrm{MHz}$.

The dipole passbands must be described using the two-chain model of Bane and Gluckstern ${ }^{4}$ as described in FNAL TM-2144. The $\mathrm{TM}_{110}$ band has $v_{0}=3979.17$ $\mathrm{MHz}$ and $\kappa=2.56 \times 10^{-9} \mathrm{MHz}^{-2}$, with the $\pi$ mode that is used for the cavity's operation at the low end of the passband, at $3900 \mathrm{MHz}$. The $\mathrm{TE}_{111}$ band has $\mathrm{v}_{0}=$ 4708.29 $\mathrm{MHz}$ and $\kappa=7.39 \times 10^{-9} \mathrm{MHz}^{-2}$, with a 0 mode at the low end of the passband.

To determine beam couplings, and to determine frequencies and beam couplings above the cut-off frequencies, numerical methods are needed. A study of HOMs using the MAFIA 2D eigensolver has calculated the frequencies and loss factors for all TM-like monopole modes up to $16 \mathrm{GHz}$ and all dipole modes up to 18 $\mathrm{GHz}^{5}$. Tables $4 \mathrm{a}$ and $4 \mathrm{~b}$, shows the results from the simulation of the cavity using the MAFIA 2D eigensolver ${ }^{6}$ for the dipole modes with the highest coupling to the beam. The beam coupling is given as $R^{(\mathrm{n})} / Q$ as defined in Table 3. Quadrupole modes do not contribute significantly to long-range wakefield effects. Prototype aluminium and copper models have basically confirmed the simulation results up to about $8 \mathrm{GHz}$.

Table 4a: Crab Cavity Eigenmode Simulation Results for Dipole Modes.

\begin{tabular}{|c|c|c|}
\hline Frequency $(\mathrm{GHz})$ & Phase Advance (degrees) & (R/Q)' (Ohms) \\
\hline 3.908 & 180 & 235.40 \\
7.082 & 160 & 2.33 \\
7.136 & 180 & 6.55 \\
7.178 & 180 & 3.62 \\
7.390 & 160 & 2.03 \\
8.039 & 20 & 4.31 \\
10.029 & 120 & 1.13 \\
10.054 & 140 & 0.73 \\
12.980 & 140 & 0.53 \\
12.996 & 120 & 1.08 \\
13.014 & 100 & 0.45 \\
17.550 & 120 & 0.15 \\
17.561 & 100 & 0.30 \\
\hline
\end{tabular}


Table 4b: Crab Cavity Eigenmode Simulation Results for Lower Order Monopole Modes.

\begin{tabular}{|c|c|c|}
\hline Frequency $(\mathrm{GHz})$ & Phase Advance (degrees) & (R/Q) (Ohms) \\
\hline 2.792 & 20 & 0.019 \\
2.796 & 40 & 0.103 \\
2.803 & 60 & 0.050 \\
2.811 & 80 & 4.786 \\
2.819 & 100 & 0.184 \\
2.827 & 120 & 115.742 \\
2.834 & 140 & 284.893 \\
2.839 & 160 & 98.614 \\
2.841 & 180 & 0.198 \\
\hline
\end{tabular}

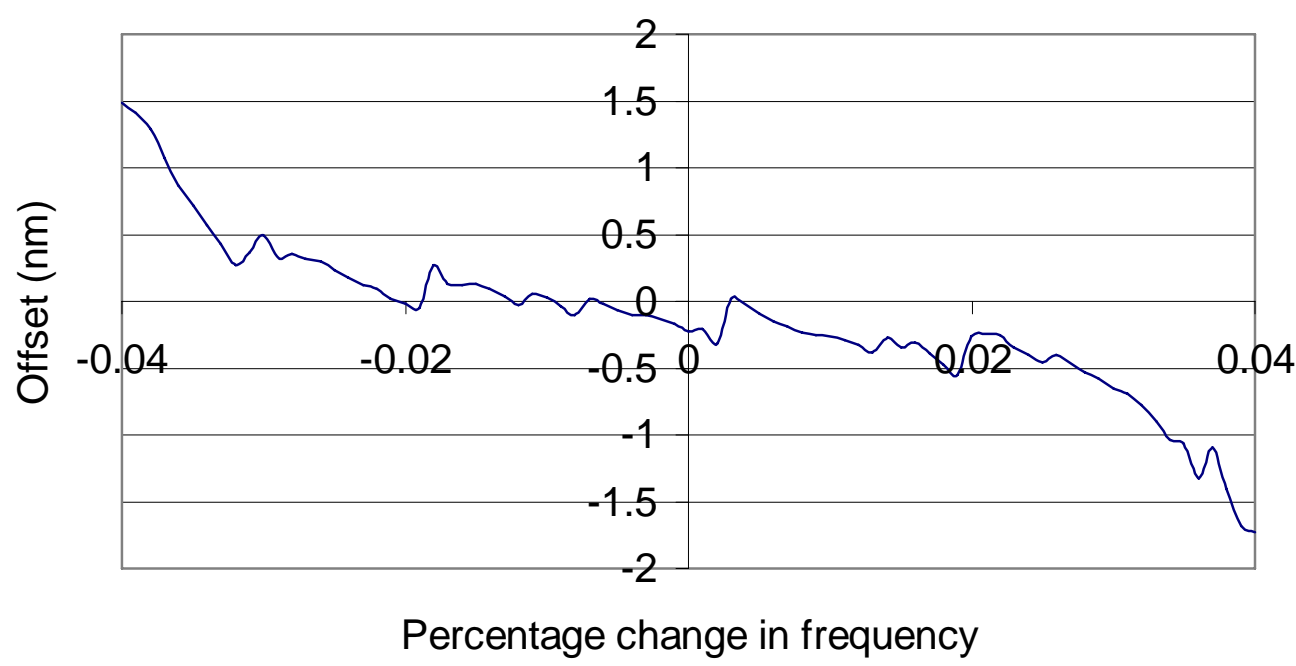

Figure 7: Vertical offset at the IP versus percentage deviation in the frequency from the nominal values calculated in MAFIA for a cavity offset by $1 \sigma_{\mathrm{y} .}$ (200 bunch are used in this simulation) calculated using PLACET

Effort is underway in inserting crab cavity long range wakefields in PLACET ${ }^{7}$ using the results given in Table 4a. A series of 200 ILC-like bunches were tracked through a single crab cavity modeling 14 dipole modes, assuming the damping of each mode ${ }^{8}$. In recent results, the vertical offset at the IP due to the crab cavity was calculated for 80 different frequencies in the range $\pm 0.04 \%$ of the MAFIA calculations, shown in Figure 7. In these simulations the Qext of the SOM (Same Order Mode) was set to the tolerance level of $2.6 \times 10^{4}$ and all other HOMs had Qext of $10^{5}$. Initial results suggest that there is little effect on beam emittance. 


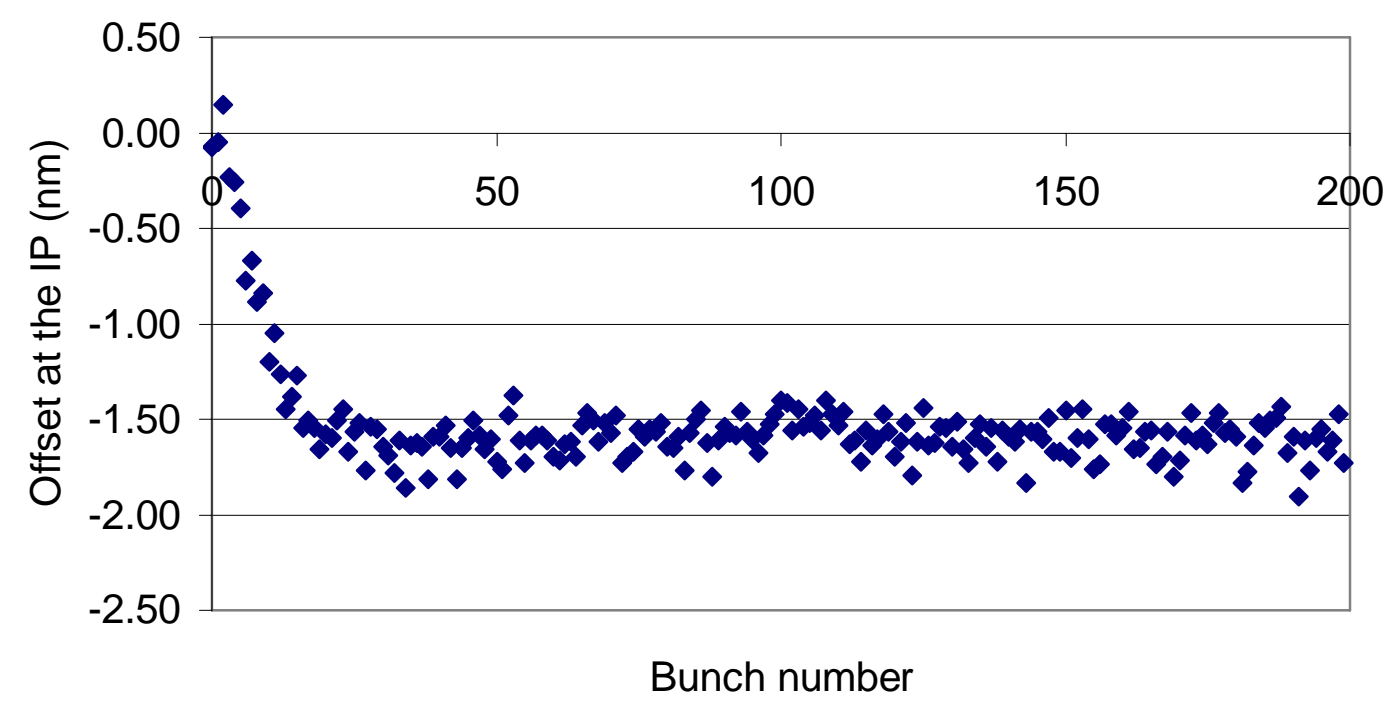

Figure 8: The vertical offset at the IP as a function of bunch number for the worst case vertical wakefields on a $250 \mathrm{GeV}$ beam, for a 30 micron vertical offset, with the SOM damped to $2.6 \times 10^{4}$ and all other modes damped to $1 \times 10^{5}$ calculated using PLACET.

The worst case scenario shows a $1.5 \mathrm{~nm}$ offset at a $-0.04 \%$ change in cavity frequencies, shown in Figure 8. The simulations include a single crab cavity and a bunch with a $500 \mathrm{GeV}$ centre of mass energy. The cavity was offset by $30 \mu \mathrm{m}$ in the simulation. Long range wakefields and their effect on the beam have also been studied using the formalism of Wanzenberg ${ }^{9}$. These calculations are found to give excellent agreement with the PLACET simulations.

The short range wakefields in the ILC crab cavity were calculated both numerically and using a combination of modal and diffractive analytical models ${ }^{10}$. The numerical model used the finite difference code ECHO 2D, which employs a moving mesh so that only the region a few millimetres behind the head of the bunch need be meshed longitudinally. This reduction in the total number of cells required allows a very dense mesh to be employed, allowing a good numerical representation of the Gaussian bunch distribution. A longitudinal mesh spacing of $10 \mu \mathrm{m}$ and a radial spacing of $100 \mu \mathrm{m}$ was used to model a bunch with a $\sigma_{\mathrm{z}}$ of $300 \mu \mathrm{m}$. Both longitudinal and transverse wakefields were identified and analysed. The loss factor for an on axis bunch was found to be $23.5 \mathrm{~V} / \mathrm{pC}$ and the transverse kick factor was found to be 69.8 $\mathrm{V} / \mathrm{pC} / \mathrm{mm}$. The analytical results are in good agreement.

There has not to date been an attempt to model manufacturing imperfections; however, several estimates have been determined using MAFIA or Microwave Studio $^{11}$ with regards to frequency changes as a function of dimensional changes ${ }^{12}$. The frequency of the cavity is lowered by $104 \mathrm{kHz}$ for every micron uniformly removed from the inner surface. Extending the equator with a cylindrical shape lowers the frequency "of the half cell" by $18.2 \mathrm{MHz} / \mathrm{mm}$. That is, if a dumbbell has 1 mm extra length on both ends, and is therefore $2 Z_{I R}+1 \mathrm{~mm}=40.4 \mathrm{~mm}$ long, it will be 3900-18.2 $=3881.8 \mathrm{MHz}$ in the $\pi$ mode. A half-cell, by itself, can not support the operating mode with any physically possible boundaries. Similarly, a cylindrical extension in the iris raises the frequency by $14.1 \mathrm{MHz}$. The frequency changes induced by a variation in each of the parameters of Table 2 are given in Table 5 . 
Table 5: Frequency Dependence Upon Cell Shape Variations.

\begin{tabular}{|l|c|c}
\hline & & $\partial \mathrm{f} / \partial \ell(\mathrm{MHz} / \mathrm{mm})$ \\
\hline half cell length & $\mathrm{g} / 2\left(\mathrm{Z}_{\mathrm{ir}}\right)$ & 17.4 \\
\hline iris radius & $\mathrm{A}\left(\mathrm{a}_{\mathrm{ir}}\right)$ & -25.6 \\
iris curvature & $\mathrm{r}_{\mathrm{i}}$ & -23.1 \\
\hline equator radius & $\mathrm{B}\left(\mathrm{a}_{\mathrm{eq}}\right)$ & -80.6 \\
equator curvature & $\mathrm{r}_{\mathrm{e}}$ & -23.2 \\
\hline
\end{tabular}

Lorentz force detuning has not been studied in detail for cavities with $2.2 \mathrm{~mm}$ wall thickness, and in any case strongly depends on the specific mechanical support structure provided for the cavity. Early studies for $1.6 \mathrm{~mm}$ thick walls found that the shift from introduction of a full-strength field is about $750 \mathrm{~Hz}$ when the cavity is supported vertically at its ends.

\section{Power Coupler}

An RF system model has been developed and utilised to verify that cavity phase can be maintained by variation of the drive phase with respect to a local reference in the presence of oscillatory beam loading and single frequency cavity vibration (microphonics) at an amplitude observed in prototype CKM cavities (see ref. 2). The full microphonic spectrum will not be known until the cryostat has been prototyped. Values of the cavity external $\mathrm{Q}$ in the range $5 \times 10^{5}$ to $10^{7}$ were considered. For this simple vibration model, it was appropriate to use a PI controller to gain insight on the level of stabilisation that might reasonably be achieved and the associated power requirement. The control loop was given a time delay of $1 \mu \mathrm{s}$. This value is optimistic for advanced controllers implemented by DSP but is easily achievable for PI controllers with analogue circuits and amplifiers with relatively low Q output stages. No measurement model was included, but the accuracy of measurement with digital phase detectors as described in the LLRF section will be a few milli-degrees. Phase measurements with passive mixers will have less jitter and the ultimate resolution will depend on bandwidth.

Figure 9 shows the cavity phase stability prediction from the model for a cavity external Q of $3 \times 10^{6}$, an oscillating beam offset of $\pm 1 \mathrm{~mm}$ at a frequency of 2 $\mathrm{kHz}$, a cavity vibration at $230 \mathrm{~Hz}$ with an amplitude that would cause a cavity frequency shift of $600 \mathrm{~Hz}$ and bunches that arrive at random times within 1 degree (at $3.9 \mathrm{GHz}$ ) of the crabbing phase. The RF system model shows that beamloading has little effect on phase stability, and hence we are able to operate using critical coupling in the presence of beam-loading at the maximum beam offset. The beam-loading does have a large effect on cavity amplitude, but fortunately the amplitude stability tolerance for the crab cavity is not very tight. At an offset of $1 \mathrm{~mm}$ the crab cavity is critically coupled at an external Q of $3 \times 10^{6}$.

Figure 9 suggests that there should be no fundamental difficulty stabilising the cavity with respect to a reference at the level of 0.01 degrees once a controller appropriate to the real microphonic spectrum has been established. For bunches arriving within $0.33 \mathrm{deg}$ (at $1.3 \mathrm{GHz}$ ) of their correct timing, the effect of beamloading on cavity phase is almost a factor of five smaller than the effect of microphonics at the level seen in prototype CKM cavities. It is anticipated therefore 
that the level of cavity phase stability that can be achieved against a local reference will be comparable with the $0.02 \mathrm{deg}$ at $1.5 \mathrm{GHz}$ (equal to a timing stability of $20 \mathrm{fs}$ ) achieved in ERLs ${ }^{13}$. The peak power requirement for the simulation of figure 9 was $250 \mathrm{~W}$ per cell or $2.25 \mathrm{~kW}$ for a nine cell cavity. If the bunch train maintains a steady offset then this peak power might be required throughout the bunch train. For an external Q of $10^{6}$ the power requirement rises to $3 \mathrm{~kW}$. For a $5 \%$ duty cycle the nominal CW power handling capacity of the power coupler is just $150 \mathrm{~W}$, although a high VSWR is expected so a higher power handling is desired. The coupler designed for the $3^{\text {rd }}$ Harmonic cavity is believed to be able to handle this level of power at $3.9 \mathrm{GHz}$, and so the crab cavity input coupler will be based around this design. Several modifications to the $3^{\text {rd }}$ harmonic coupler are required to adapt it to the crab cavity, due to the smaller beampipe radius and different external Q requirements.

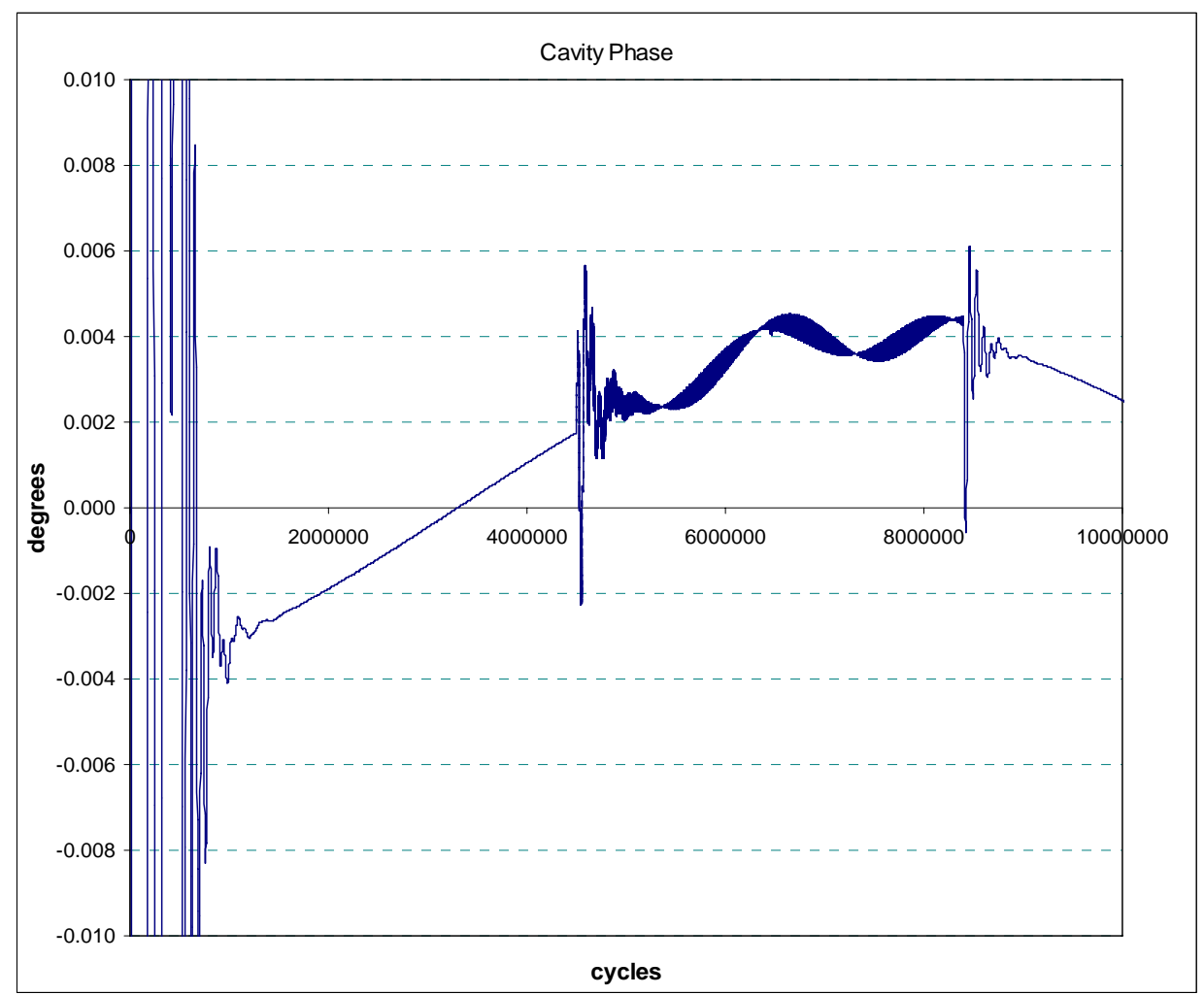

Figure 9: Cavity Phase for Oscillating Beam Offset \& Microphonics $Q_{e}=3 \times 10^{6}$

\section{Lower Order Mode (LOM) Coupler}

The monopole mode of the cavity resonates at a much lower frequency than the operating mode. This mode has a very high loss factor and does not couple strongly to traditional couplers. A special hook-type coupler is required to effectively damp the modes in the first monopole passband. Due to the high loss factor of the $7 \pi / 9$ monopole mode, a large amount of energy may be deposited into the cavity by the beam if the bunch-train hits the resonance of this mode. To reduce the average power flow in the LOM coupler below 5 Watts, the required $Q_{\mathrm{EXT}}$ for this mode must be $1 \times 10^{4}$ or less. However it may be possible to increase the average power flow limits by using a carefully designed coaxial waveguide to remove the LOM power out of the cryomodule. If the power flow can be increased to 30 Watts an external Q of 
$10^{5}$ would be required, which is readily achievable with current designs of the LOM coupler.

The LOM coupler design for the crab cavity is based on the design for the FNAL CKM cavity. A few modifications were incorporated in the $\operatorname{design}^{14}$ to improve its mode coupling performance. The most significant modification is the removing of the $3.9 \mathrm{GHz}$ notch filter by placing the LOM coupler on the same (vertical) plane as the SOM coupler by taking the advantage of the natural filter formed by the polarization symmetry. This modification simplifies the geometry and improves the damping. A comparison of the $Q_{\mathrm{ExT}}$ between the original design and the new design is shown in Figure 10. The overall $Q_{\mathrm{EXT}}$ for the monopole band is lower with the new design and the $Q_{\mathrm{EXT}}$ is below $1 \times 10^{5}$ for the $7 \pi / 9$ mode, which gives an average power flow of 30 Watts.
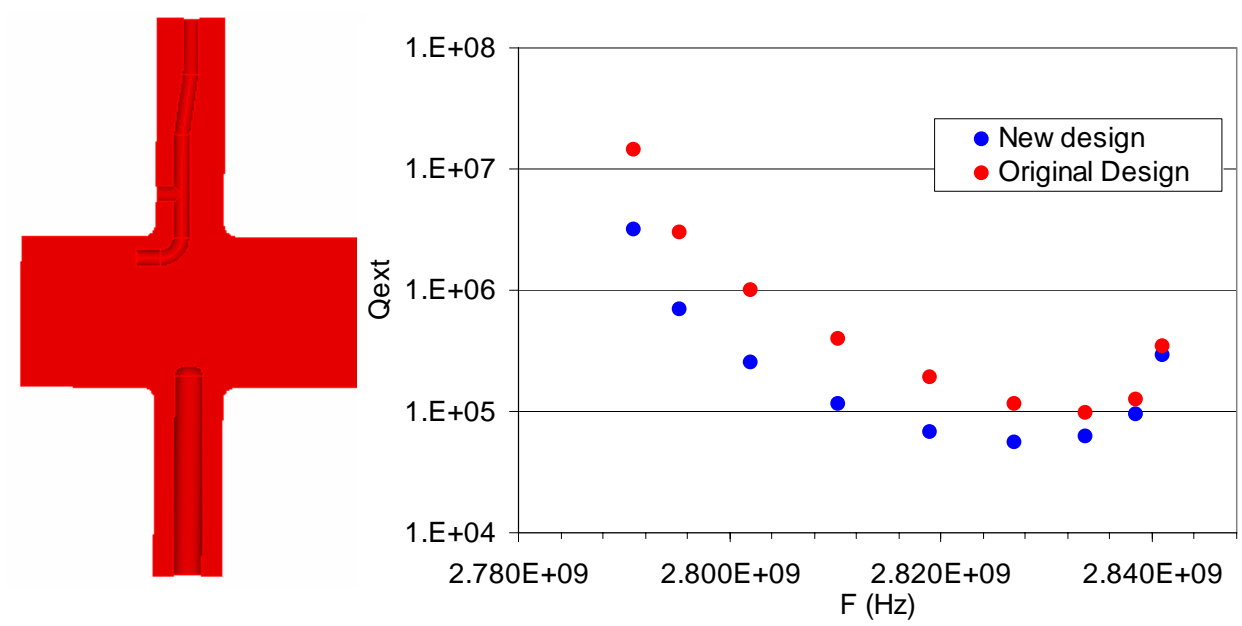

Figure 10: The new LOM coupler and calculated Qext for the 1st monopole band.

\section{Same Order Mode (SOM) Coupler}

The same order mode is the alternative polarisation of the operating mode. As the operating mode has been designed to have a high shunt impedance, the SOM will also have a high shunt impedance. As the SOM is only $10 \mathrm{MHz}$ away from the operating mode a special coupler is required to damp this mode without coupling to the operating mode. Simulations using Omega3-P have found that this mode is coupled to the $7 \pi / 9$ mode of the opposite polarisation as shown in Figure 11. This coupling causes a twisted field distribution which induces $\mathrm{x}-\mathrm{y}$ coupling and reduces the effectiveness of the SOM coupler; hence this coupling could be problematic. Minor modifications to the cavity geometry are envisioned to remove this coupling by altering the frequencies of these modes.

The required damping of the SOM, if it is on resonance with the beam, is $2.6 \mathrm{x}$ $10^{4}$. We hope to tune the cavity such that the SOM is not on resonance, however manufacturing errors may cause the modal frequency to shift hence we would like to achieve this specification as a precaution. There are currently two designs under development for this coupler. The first design is a modified input coupler with active damping as shown in Figure 12. A $Q_{\mathrm{EXT}}$ of $4 \times 10^{5}$ can be achieved with an intrusion 
of 3mm. Results at Jlab ${ }^{15}$ have shown that active damping can reduce the external Q of a single mode using a feedback loop. This design uses a phase control system to decrease the loaded $\mathrm{Q}$ by more than an order of magnitude allowing the external $\mathrm{Q}$ to be reduced to $2.6 \times 10^{4}$. The second design uses the LOM coupler to damp both the SOM and the LOM modes, which would allow for the elimination of the SOM coupler, simplifying the geometry and saving cost. This arrangement allows for the coupler to be directly adapted to a coax cable, without the notch filter tuning gap that is common to many coupler designs. The optimization of such a design is in progress.
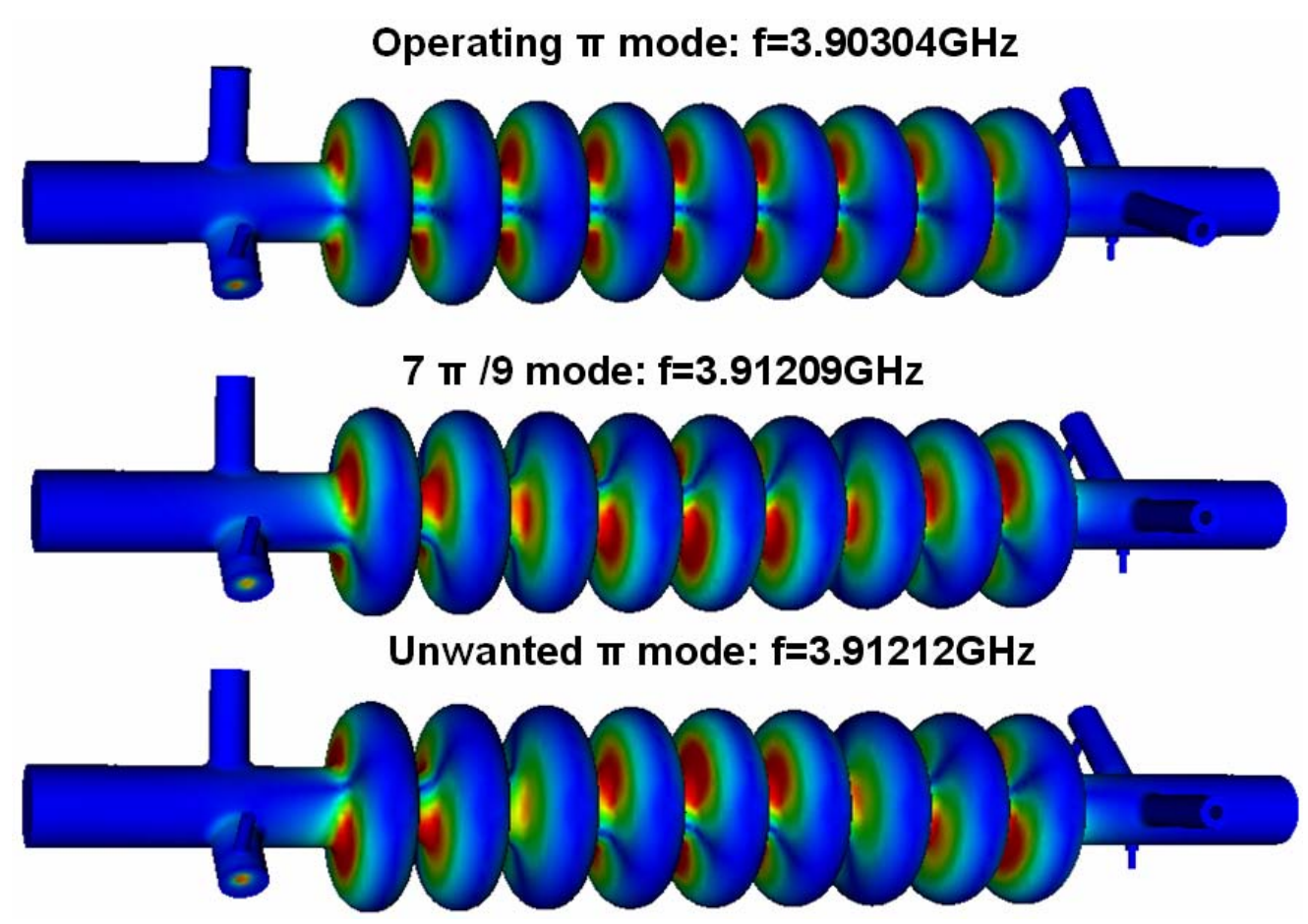

Figure 11: The twisted modes in the cavity when mode coupling exists. The twisted field distribution induces $x-y$ coupling and reduces the effectiveness of SOM damping. This mode coupling is removed by a minor modification to the cell shape.

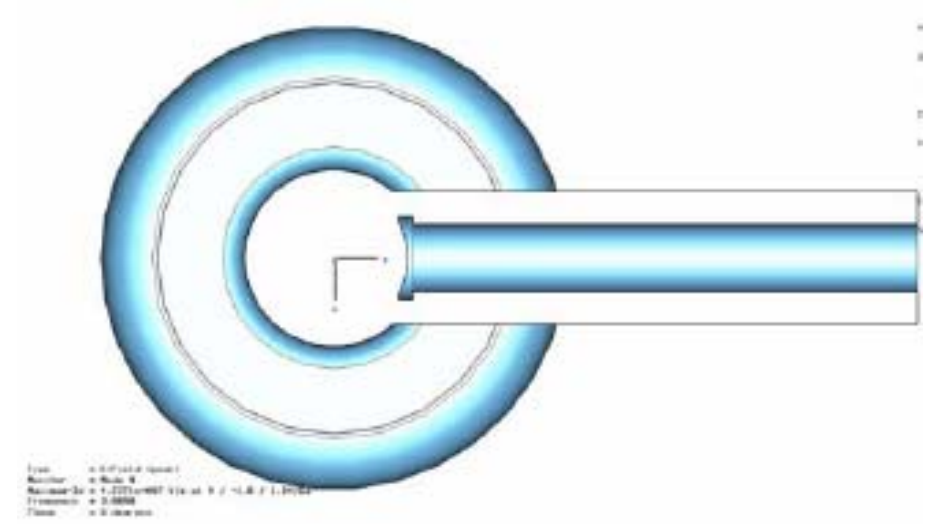

Figure 12: A SOM coupler design similar to the input coupler. This design can damp the SOM mode to below $2.6 \times 10^{4} Q_{\text {EXT }}$ with active damping. 


\section{Higher Order Mode (HOM) Coupler}

The requirements for the damping of monopole and dipole modes have been calculated for the ILC crab cavity. The damping required for some of the most dangerous modes is given in Table 6 . The requirements for modes other than the LOM and SOM do not seem to be a challenge. Because of the high incident energy of the beam, the dispersion introduced by monopole modes is a secondary concern.

Table 6: Required Damping for the LOM and Dipole Modes.

\begin{tabular}{|c|c|c|}
\hline Mode Order & Frequency $(\mathrm{MHz})$ & External Q \\
\hline Monopole & 2792 & $1.00 \mathrm{E}+08$ \\
Monopole & 2796 & $1.00 \mathrm{E}+07$ \\
Monopole & 2803 & $1.00 \mathrm{E}+07$ \\
Monopole & 2811 & $2.00 \mathrm{E}+05$ \\
Monopole & 2819 & $1.00 \mathrm{E}+07$ \\
Monopole & 2827 & $2.00 \mathrm{E}+04$ \\
Monopole & 2834 & $1.00 \mathrm{E}+06$ \\
Monopole & 2839 & $1.00 \mathrm{E}+07$ \\
Monopole & 2841 & $1.00 \mathrm{E}+09$ \\
Dipole & 3912 & $2.63 \mathrm{E}+04$ \\
Dipole & 7082 & $1.46 \mathrm{E}+06$ \\
Dipole & 7136 & $5.14 \mathrm{E}+05$ \\
Dipole & 7178 & $7.28 \mathrm{E}+05$ \\
Dipole & 7390 & $1.60 \mathrm{E}+06$ \\
Dipole & 8039 & $6.90 \mathrm{E}+05$ \\
Dipole & 10029 & $2.12 \mathrm{E}+06$ \\
Dipole & 10054 & $2.86 \mathrm{E}+06$ \\
Dipole & 12980 & $3.49 \mathrm{E}+06$ \\
Dipole & 12996 & $1.71 \mathrm{E}+06$ \\
Dipole & 13014 & $2.81 \mathrm{E}+06$ \\
Dipole & 17533 & $8.19 \mathrm{E}+06$ \\
Dipole & 17541 & $4.55 \mathrm{E}+06$ \\
\hline
\end{tabular}

The experience gained in developing the HOM couplers for the $3^{\text {rd }}$ Harmonic cavity can be directly used for the crab cavity. A modified version of the HOM coupler based on an older version of the $3^{\text {rd }}$ Harmonic cavity design has been optimized at SLAC to improve the notch filter sensitivity and Qext damping. In the new design, the notch gap is increased to 3.1-mm which results in a notch tuning sensitivity of about $0.1 \mathrm{MHz} / \mu \mathrm{m}$ (shown in Figure 13), as compared with the 1.6 $\mathrm{MHz} / \mu \mathrm{m}$ notch gap adjustment in the original design. The loop shape of the antenna is replaced with a two-stub probe antenna. The coupler is placed in the horizontal plane to damp the horizontal HOM modes, which also preserves the $\mathrm{x}-\mathrm{y}$ symmetry. The HOM modes in the vertical polarization are damped primarily by the SOM coupler. The Qext of the first and the second dipole bands is shown in Figure 14. Because of the notch filter of the HOM coupler at $3.9 \mathrm{GHz}$, the $Q_{\text {EXT }}$ of the operating 
mode (the solid circle) is determined by the input coupler. The solid triangle shows the $Q_{\mathrm{EXT}}$ of the SOM mode ( $\pi$ mode) which is calculated using the original SOM coupler design.
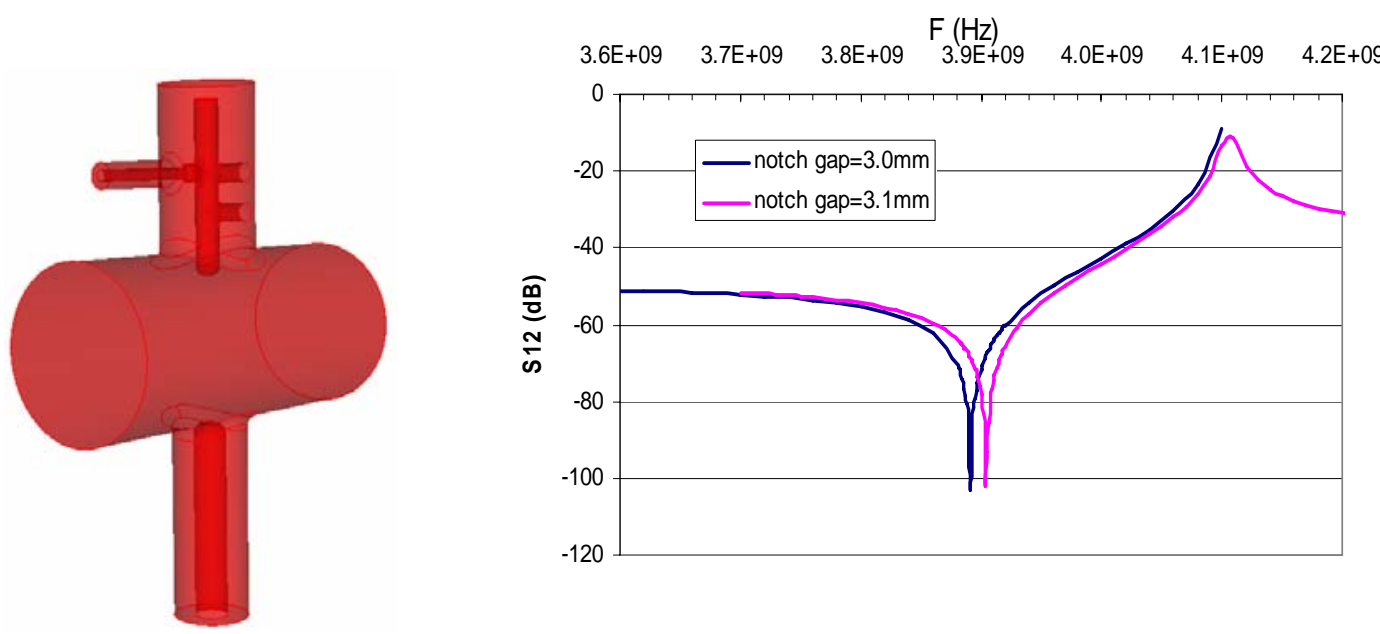

Figure 13: HOM coupler and and notch filter sensitivity. A 0.1-MHz/micron notch sensitivity achieved with the new design

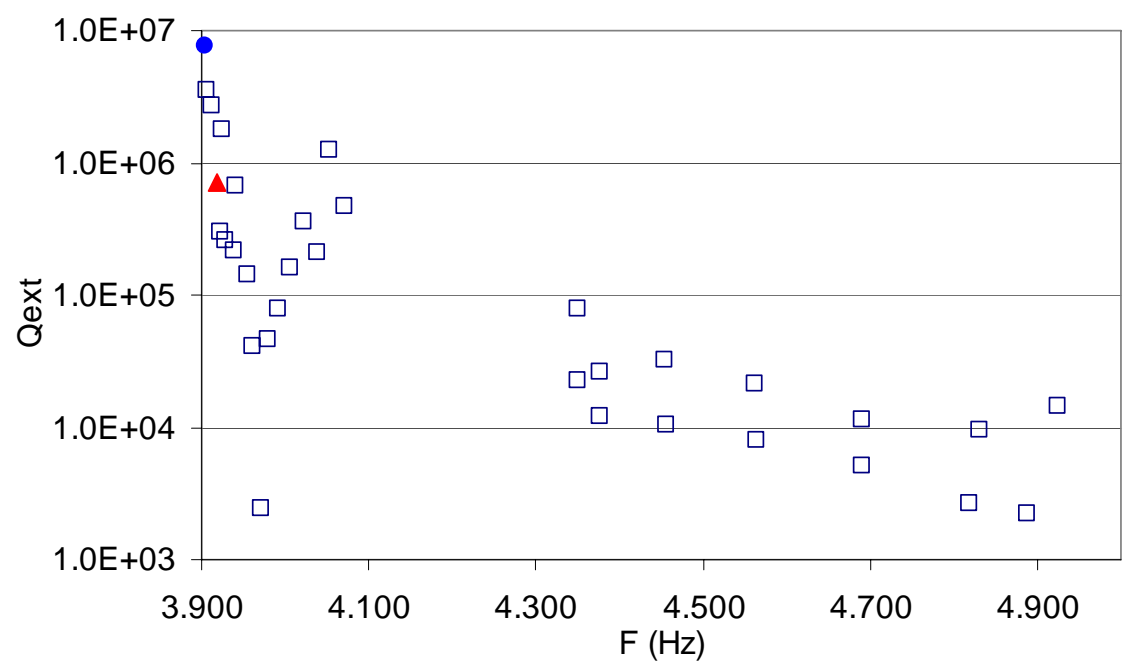

Figure14: HOM damping of the first and second dipole bands. The solid circle is the operating mode. The solid triangle is the pi-mode of the opposite polarization.

\section{Phase and Amplitude Tolerances}

The crab cavity phase must be controlled to a tight tolerance to avoid large centre of mass kicks to the bunch. Table 7 shows the results of a Monte Carlo study ${ }^{16}$ for a $250 \mathrm{GeV}$ beam with $14 \mathrm{mrad}$ crossing angle. Analytical results based on geometric arguments give good agreement with these results ${ }^{17}$. This specification is 
driven by the narrow width of the bunch at the IP relative to the effective distance of the crab systems from the IP.

Table 7: Tolerances for crab cavity timing and amplitude control, and beam timing and energy for $250 \mathrm{GeV}$ beam with $14 \mathrm{mrad}$ crossing angle. Considered separately, each of these tolerances will limit the average luminosity to $<2 \%$.

\begin{tabular}{|c|c|c|}
\hline & $\begin{array}{c}\text { timing(phase) tolerance } \\
\text { limiting luminosity loss to } \\
<2 \%\end{array}$ & $\begin{array}{c}\text { amplitude tolerance } \\
\text { limiting luminosity loss to } \\
<2 \%(\%)\end{array}$ \\
\hline RMS beam timing jitter & $0.68 \mathrm{ps}=0.95^{\circ}$ & 0.33 \\
\hline $\begin{array}{c}\text { RMS beam energy jitter } \\
\text { RMS cavity timing jitter } \\
\text { (uncorrelated) }\end{array}$ & $0.067 \mathrm{ps}=0.094^{\circ}$ & \\
\hline $\begin{array}{c}\text { RMS cavity timing } \\
\text { jitter(anticorrelated) }\end{array}$ & $0.047 \mathrm{ps}=0.066^{\circ}$ & 6.6 \\
\hline RMS cavity amplitude jitter & & \\
\hline
\end{tabular}

In these simulations the phase difference between each cavity and a perfect reference was modeled, and this is how the numbers of Table 7 are to be interpreted. In the ILC crab system one cavity will be used as a phase reference for the other. This increases the permissible timing jitter tolerance by a factor of $\sqrt{2}$, giving an intercavity timing (phase) jitter tolerance of 95 fs $\left(0.133^{\circ}\right)$.

This is a very tight specification; for comparison, the JAERI ERL-FEL RF control system is able to maintain $0.1^{\circ}$ at $0.4498 \mathrm{GHz}$, or $618 \mathrm{fs}$; a 2005 DESY report gives $0.1^{\circ}$ at $1.3 \mathrm{GHZ}$, or $213 \mathrm{fs}$ as the required scale of control needed for the VUVFEL Linac. ${ }^{18}$

Beam loading issues are closely intertwined with the RF control system. When the beam enters the cavity off-axis in the horizontal plane, it will either insert or remove energy from the cavity. The LLRF and beam control feedback systems will correct for this, but will function better as we are able to develop couplers of lower $Q_{\text {EXT }}$ and greater power handling capability.

\section{LLRF Prototype Tests}

Components for the type of LLRF system envisaged are relatively inexpensive and indeed new superior components become available each year. There are a wide range of possible layouts and technologies for the LLRF system and details of the systems described here are likely to be superseded before the ILC starts collecting data. Here we set out two schemes that are currently under development. The first is relatively straight-forward to implement and has the potential to just meet the demanding phase synchronisation and stabilisation requirements for the ILC crab cavities. The second is more difficult to implement but has the potential to exceed 
these requirements. A hybrid of the two systems is a likely final scenario. By stabilisation we mean controlling the phase jitter of the cavity arising from microphonics with respect to a local reference. By synchronisation we mean providing synchronous reference signals at the cavity locations.

The objective for the low level RF system is to synchronise the phase of two RF cavity systems to better than $0.1^{\circ}$ at $3.9 \mathrm{GHz}$. This corresponds to a timing synchronisation of $71 \mathrm{fs}$ and for a light signal a corresponding wavelength of $21 \mu \mathrm{m}$.

Given the relative low cost of the LLRF system, it is not unreasonable to have separate control circuits for each cavity and separate synchronisation circuits between symmetrical pairs of cavities. On this basis we only consider the control and synchronisation of one symmetrically positioned pair of crab cavities (one on the positron beam line and the other on the electron beam line).

Making assumptions on the maximum level of microphonics and beam loading from offset bunches, the power requirement for each individual 9-cell cavity with an $Q_{\text {EXT }}$ of $3 \times 10^{6}$ is about $3 \mathrm{~kW}$. This power level is low enough that it can be delivered by commercially available solid state amplifiers. Manufacturers currently have designs for $2 \mathrm{~kW}$ at $3.9 \mathrm{GHz}$. Commercially available klystrons can supply from $5-90 \mathrm{~kW}$ at this frequency and hence if for operational reasons it was necessary to operate with a lower $Q_{\mathrm{EXT}}$ then there is no difficulty with supplying sufficient power using these devices. It should be noted that for a crab cavity, beam-loading acts to perturb the amplitude rather than the phase, unless the bunch timing errors are large compared to the ILC specification. Microphonics perturb the phase.

Interestingly the precise arrival time of the bunches is not as critical as the synchronisation of the cavities. However the longitudinal position of the cavities is just as critical as the synchronisation. The longitudinal positions must therefore be stable to much better than $21 \mu \mathrm{m}$. In principle the cavity synchronisation could be varied to compensate for longitudinal movement of the cavities however this is not considered here.

The planned schemes each use an RF interferometer to provide synchronous reference signals. The ends of the interferometer will utilise phase detectors positioned very close to cavity output couplers. The input to the interferometer phase detectors which are made synchronous by control action are split to give a reference input to cavity phase detectors as shown for scheme 1 in Figure 15 . The interferometer and cavity phase detectors are on the left and right respectively. The interferometer phase detector at each end will be mounted adjacent to the associated cavity phase detector on the same PCB and at an electrical separation of half of a wavelength so that any small reflected signals perturb the amplitude rather than the phase at the other detector.

The performance of the overall system depends critically on the performance of the phase detectors. Many current accelerator LLRF systems use double balanced mixers in quadrature to mix down the signal thereby allowing phase and amplitude to be measured accurately on a low frequency signal. Where the signals are sampled digitally, the measurement of phase depends critically on the accuracy to which a clock in the processor can be established. As this type of a scheme is being developed for the main linac, its use for the crab cavity is also a possibility along with other clock based schemes. 


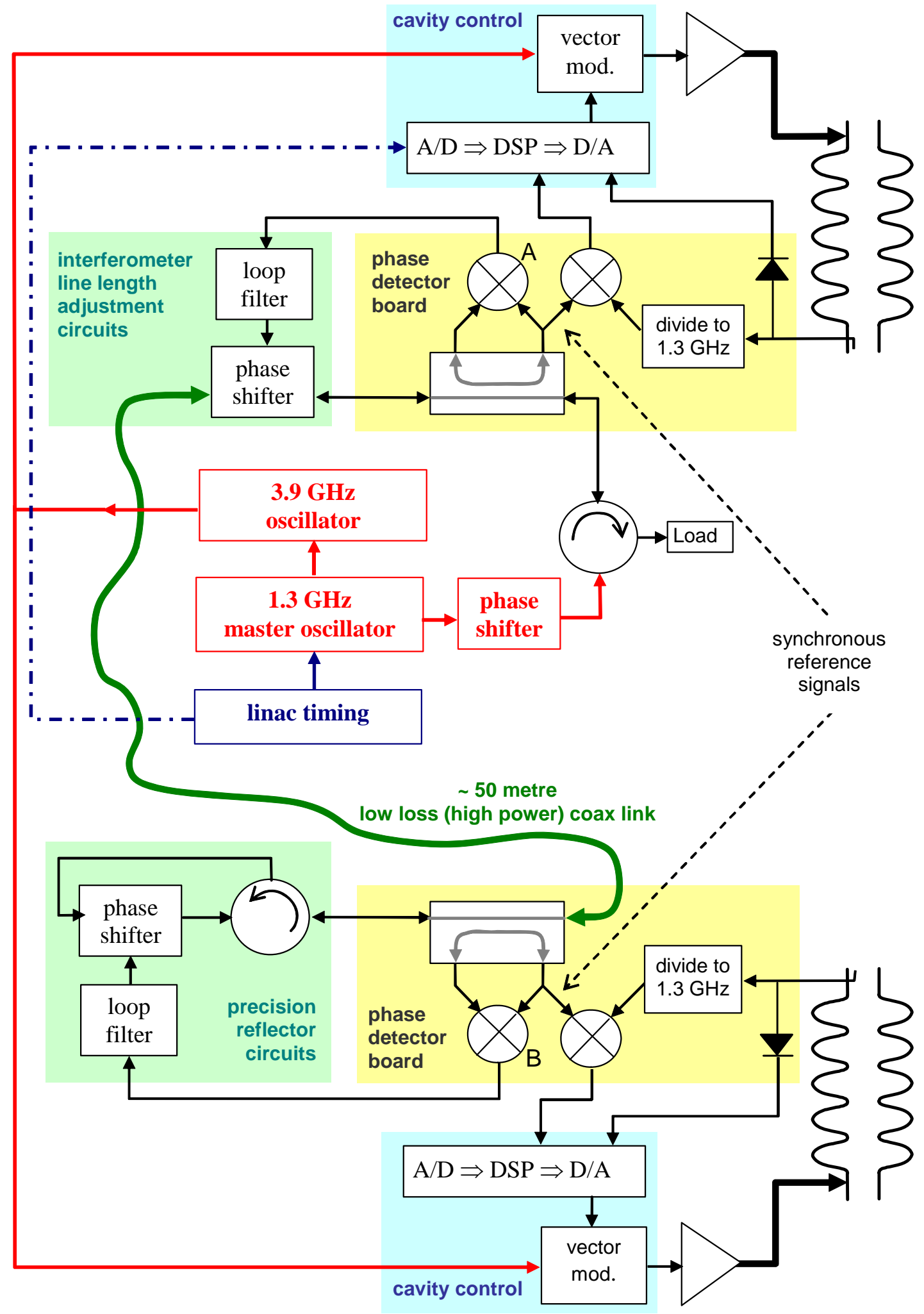

Figure 15: LLRF Scheme 1 (Scheme 2 operates at $3.9 \mathrm{GHz}$ throughout)

The first system currently under investigation specifically for the crab cavity uses HMC439 digital phase detectors. The digital phase detector gives an analogue d.c. voltage output in proportion to the phase difference of the input from a reference. 
These phase detectors operate at frequencies up to $1.3 \mathrm{GHz}$ and have an r.m.s. jitter of 5 milli-degrees for an output band width of $1 \mathrm{MHz}$.

The advantage of using digital phase detectors is that they have no sensitivity to the amplitude of the input signal and give an absolute phase measurement of $\pm 180^{\circ}$ either side of zero without calibration. Consequently they are simple to implement within a phase lock loop.

The second scheme to be investigated will use passive mixers with lower phase noise than the digital mixers. For this scheme and cavity and interferometer phase measurements will be made at $3.9 \mathrm{GHz}$.

The electrical length of the interferometer is controlled by a phase lock loop on the upper circuits of Figure 15 which acts on a phase shifter. The termination/reference point is the centre of the digital phase detector A. The effective point from where the signal is reflected is the centre of phase detector $\mathrm{B}$. The phase lock loop on the lower circuits control the phase of the wave which returns. Circuitry for the interferometer is semi-analogue for scheme 1 and fully analogue for scheme 2 and hence will react to changes in the line length on as short a timescale as necessary. This timescale is determined by the loop filter which acts as the controller. The interferometer can in principle lock the signal at $\mathrm{A}$ and $\mathrm{B}$ to a difference of either $0^{\circ}$ or $180^{\circ}$. The line length in conjunction with the range of the phase shifter can be chosen to ensure that the signals are always locked to $0^{\circ}$. For scheme 1 the interferometer will operate at $1.3 \mathrm{GHz}$ as digital phase detectors are not currently available at higher frequencies.

For scheme 1, the phase lock loop on the interferometer will operate with a bandwidth less that $100 \mathrm{kHz}$ and hence the 5 milli-degrees of jitter at $1.3 \mathrm{GHz}$ for $1 \mathrm{MHz}$ bandwidth will be exceeded. Measurements suggest that 3 milli-degrees will be achieved with this bandwidth and hence the interferometer is expected to have an r.m.s. phase jitter of less than 4.3 milli-degrees at $1.3 \mathrm{GHz}$ which is equivalent to 13 milli-degrees at $3.9 \mathrm{GHz}$. If synchronisation to this level is possible, then to achieve overall synchronisation between the cavities of 100 milli-degrees the cavity stabilisation circuits must each control jitter to 43 milli-degrees which is equivalent to 14 milli-degrees at $1.3 \mathrm{GHz}$.

For scheme 2, the phase locked loop on the interferometer will operate at $3.9 \mathrm{GHz}$. The phase noise of the passive mixers will be much less than that of the digital phase detectors. Cavity phase will also be measured at $3.9 \mathrm{GHz}$ hence the summed measurement uncertainty will be a small fraction of the phase tolerance required. A difficulty that arises with the passive mixers in a phase locked loop is that they have their maximum sensitivity when the input and reference signals are $90^{\circ}$ apart and can only measure over a range of $180^{\circ}$. The offsets for the two interferometer mixers combine so that there is an uncertainty in the phase of $180^{\circ}$. Unlike the previous case, this phase uncertainty is independent of the length of the interferometer. A second difficulty is that the measured phase depends on amplitude and hence while the cavity is being filled, control is more difficult. A third difficulty arises when one cavity has to be operated at a small calibrated phase offset from zero. All of these difficulties are readily resolved if digital phase detectors are used alongside the policing action of the passive mixers. Such a hybrid scheme is likely to yield the best overall performance. 
Control algorithms for the cavity phase stabilisation will be implemented with a digital signal processor. As SRF cavities are operated with high $\mathrm{Q}_{\mathrm{e}}$ there is a considerable delay between the measurement of a phase error and its correction using analogue control. Intelligent control allows prediction of necessary control action in advance of a measurable error. Stabilisation of cavities using intelligent control to the level of 20 milli-degrees at $1.3 \mathrm{GHz}$ has been demonstrated elsewhere ${ }^{13}$. Optimisation of intelligent control for the ILC crab cavities cannot be completed until fully developed cavities and horizontal cryostats are available.

For scheme 1, the cavity operates at $3.9 \mathrm{GHz}$ whilst phase detection operates at $1.3 \mathrm{GHz}$. Consequently, the output signal must be divided by 3 and hence the phase error gets divided by 3 as well. Jitter for the dividers is an order of magnitude smaller than that for the phase detectors hence for a phase detector jitter of 5 millidegrees we have a resolution of 15 milli-degrees at $3.9 \mathrm{GHz}$. The cavity controller must therefore maintain the cavity phase within 9 milli-degrees of the measured phase at $1.3 \mathrm{GHz}$ for the required phase synchronisation to be achieved.

The anticipated challenge with the interferometer is not so much the jitter but the management of systematic errors caused by reflection at component interfaces. A reflected power of just $-40 \mathrm{~dB}$ corresponds to $1 \%$ reflected voltage and could potentially give rise to a $1^{\circ}$ phase error if this reflection has a phase shift of $\pm 90^{\circ}$ when it arrives back at a phase detector. In order to minimise these systematic errors all signal pathways must be made close to an exact number of half wavelengths and as a consequence there may be a requirement to thermally stabilise the interferometer. A detailed model of spurious reflections within the interferometer is still required. It is expected that the net error will be steady and hence compensation can be made after calibration with a luminosity scan.

In the analysis to this point no inclusion of variation in path length between the measurement point and the cavity centre of kick has been made. If variation of this path length is less than 1 micron, then taking both systems into account, the phase tolerance is only stretched at worst by an additional $10 \%$.

Current plans for the digital control circuits utilise 16 bit ADCs with a sample rate of 100 MSPS, a latency of 130 ns and r.m.s. noise of 2.4 bits. Nominally 16 bits gives a measurement resolution of 5.6 milli-degrees in 360 degrees at $1.3 \mathrm{GHz}$ but with 2.4 bits of noise this is reduced by a factor of 5.3. In order to avoid averaging, the output from the digital phase detector will be split and amplified so that one output measures $0^{\circ}$ to $360^{\circ}$ with a resolution of about 30 milli-degrees and the other output measures a range of $\pm 5^{\circ}$ with a resolution slightly better than 1 milli-degrees. Test boards will have six inputs to the DSP used as follows, two for outputs from the digital phase detector, one for the amplitude diode detector, one for linac timing signals, one reporting information from the piezo tuners and one spare. During development, performance of the digital phase detectors will be compared to performance from double balanced mixer used in quadrature. For this arrangement the spare input together with the input for the diode or the input for the piezo tuner will be utilised.

Data from the ADCs will be written directly to the DSP memory by FPGAs with a latency of about 50 ns. Processing by the DSP will take about 1000 ns. Corrections to the drive phase and amplitude are made with a vector modulator taking IQ inputs. The DACs providing these input signals from the DSP have a settling time of $10 \mathrm{~ns}$. Overall the control system has the ability to react to measurement errors on a time scale of $1.5 \mu \mathrm{s}$. 
A critical test is planned for the LLRF system in Q2 2007 by evaluating the control of two matched SRF cavities in a vertical cryostat. Testing of cavity stabilisation performance will ultimately require a fully developed cavity in a full integrated cryostat with beam. Understanding phase jitter between the centre of kick and the phase detector will need a beam line with two cavities operated in deflection mode.

\section{Cavity Ancillaries}

A prototype cold tuner, shown in Figure 16 has been assembled and undergone preliminary testing at FNAL. It has a very fine motor control - nominally, 6.4nm per stepper motor step - and at room temperature shifts the frequency of a 13-cell cavity with wall thickness $1.6 \mathrm{~mm}$ at a rate of $1.048 \pm 0.020 \mathrm{MHz} / \mathrm{mm}$. The tuner design does have space for a piezoelectric element to be inserted so that it may operate in concert with the stepper motor. The design shown is for a LHe cryovessel of 6 inch diameter; note that the blade tuner design of the $3{ }^{\text {rd }}$ Harmonic effort fits around a LHe vessel of 5 inch diameter and the cavity itself is close to a 4 inch diameter. Accordingly, if adequate cryogenic stability can be maintained with this volume of LHe, the $3^{\text {rd }}$ Harmonic tuner could probably also be used.

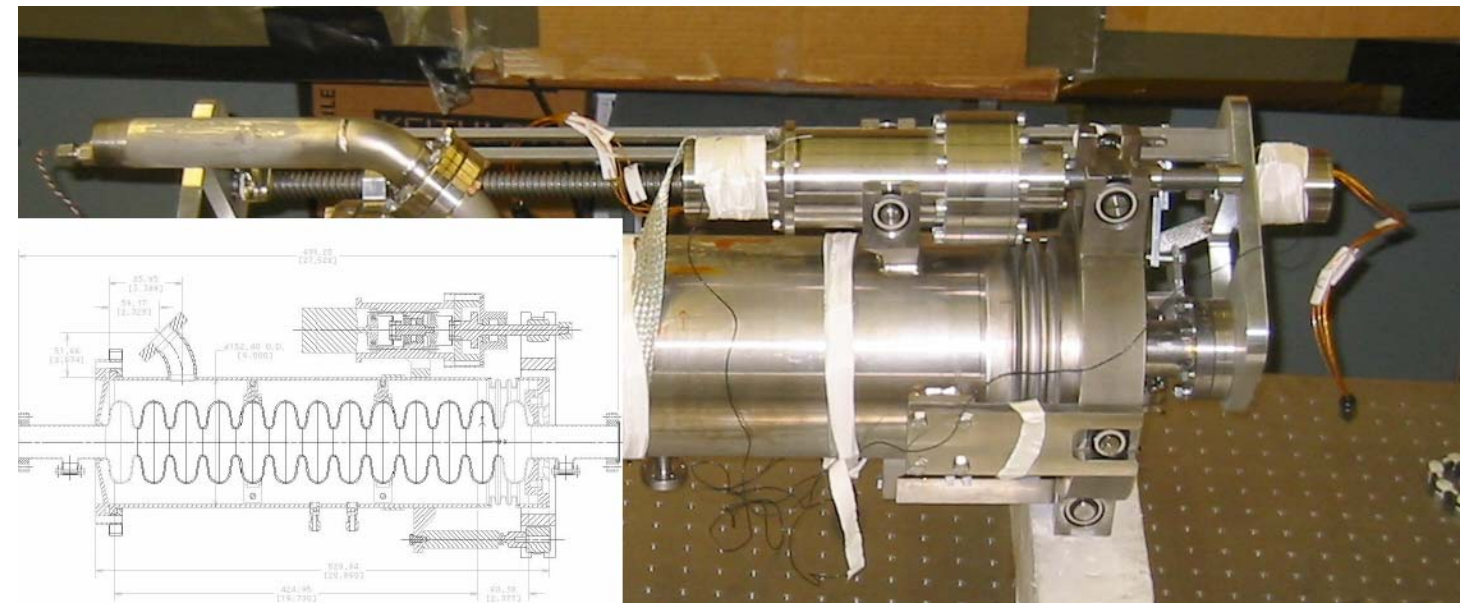

Figure 16: Prototype cold tuner; inset, mechanical drawing of same.

\section{Cavity Positioning}

In order to reduce the transverse wakefield induced in the cavity, it is necessary to keep the beam as close to the centre of the cavity as possible. The dipole HOMs in the vertical plane are exceptionally sensitive to transverse offsets, and hence the positioning of the cavity must be controlled to within $\sim 15 \mu \mathrm{m}$. The longitudinal positioning requirement of $21 \mu \mathrm{m}$ has been discussed above in the context of the LLRF system. If this requirement is not met, additional damping from the SOM coupler will be required to account for the higher loss factor of this mode. Final adjustment can be done by adjusting the cavity position to minimise the signal from the SOM coupler. 


\section{Field Polarisation (Roll) Tuner}

The ideal crab cavity would provide a horizontal crabbing effect to the bunch and would have no effect in the vertical plane. However, if the polarisation of the operating mode is not aligned exactly in the horizontal plane there will be a spurious crabbing effect in the vertical plane as well. As the vertical beam size is so small the polarisation of the crabbing effect must be controlled to less than $1 \mathrm{mrad}$. This will require the development of a roll tuning mechanism to rotate the polarisation of the crabbing field. In order to measure the polarisation a dipole magnet will be used to cancel the field in the horizontal plane and the vertical kick in deflecting phase will be measured. The polarisation of the dipole magnet can be measured using lasers.

\section{Cryostat}

A cryostat with suitable mechanical stability and adjustability needs to be designed. The design must also provide microphonic rejection such that the LLRF can achieve the required phase control, and it must also provide enough mid-cavity support to keep the alignment of electrical centres of the cells from becoming an issue.

The extraction beamline is located $18.7 \mathrm{~cm}$ from the centre of the cavity at its closest point. The cryostat design must take this extraction beamline into account.

\section{Beam Testing and Validation}

Beam testing and validation of the proposed crab cavity and phase control systems is paramount in determining the achievable performance on ILC. The planned beam tests on ILCTA at FNAL can be characterised into two parts: cavity tests based on a single crab cavity and tests based on a two cryostat crab cavity system. All of these tests can be carried using a $40 \mathrm{MeV}$ beam tapped off from the main injection line.

With a single crab cavity, wakefields may be measured with an unpowered cavity. (All the other tests require that the cavities be fully powered). Tests of bunch longitudinal size and shape, and measurements of associated arrival time jitter will be carried out. An independent EO bunch arrival time monitor and longitudinal profile monitor is proposed to facilitate such tests. Beam loading, coupler power loading and microphonics will also be investigated.

The complete crab cavity test system, comprising two adjacent crab cavities powered through a phase reference distribution or synchronisation system, is the full test that shows that the crucial phase control specification has been met under realistic conditions. The 'crabbing' field polarisation can also be verified with a crab cavity installed in a cryovessel with a suitable roll tuner.

\section{Conclusion}

The ILC Crab Cavity is proposed to be a 9-cell superconducting RF cavity operating at $3.9 \mathrm{GHz}$ based on the FNAL deflecting mode cavity. A 3-cell prototype of this cavity has been manufactured and achieved a gradient of $7.5 \mathrm{MV} / \mathrm{m}$ in cold tests. 
The impedance of this cavity has been studied in depth. Large beam-cavity interactions occur in the other-polarization same-order mode. The effects of these impedances on the beam in the ILC beam delivery system have been calculated analytically and simulated using PLACET in order to calculate damping tolerances on the modes of the cavity. Three couplers have been designed in order to meet these damping tolerances, one for the lower-order modes, one for the same-order modes and one for the higher-order modes. The same-order mode appears to require active damping in order to meet its damping requirement.

The phase and amplitude stability requirements have been studied both analytically and numerically. An uncorrelated timing jitter of $67 \mathrm{fs}$ RMS must be achieved and the amplitude stability must be kept to better than $6.6 \%$ to keep the luminosity loss below 2\%. A DSP based system will be used to control the phase and amplitude at each cavity, and an RF interferometer is proposed in order to synchronize both cavities.

\section{Acknowledgements}

This work is supported by LC-ABD grant number PP/B500007/1., by the Commission of European Communities under the FP6 "Research Infrasctructure Action - Structuring the European Research Area” EUROTeV DS Project Contract no.011899 RIDS, by PPARC and the U.S. Dept. of Energy contract number DEAC02-76SF00515 and by FRA/DoE contract number DE-AC02-07CH11359

\section{References}

${ }^{1}$ International linear Collider Reference Design Report (Feb 2007), [online] http://media.linearcollider.org/rdr_draft_v1.pdf..

${ }^{2}$ D.Edwards, Editor, FNAL TM-2060, November 1998; M.McAshan and

R.Wanzenberg, TM-2144, March 2001

${ }^{3}$ L.Bellantoni, H.Edwards, T.Khabibouline, A.Rowe, Field Flatness Tuning of $\mathrm{TM}_{110}$ Mode Cavities With Closely Space Modes, SRF 2003, 8-12 Sept. 2003, Travemunde, Germany.

${ }^{4}$ K.L.F. Bane and R.L. Gluckstern, SLAC-PUB-5783, March 1992. The extention to include resistive elements is straightforward, and the ratio of cell amplitudes in the last cell over amplitudes in the first cell is capable of providing a qualitative model of $S_{12}$. (L. Bellantoni, M. Astner, unpublished).

${ }^{5}$ G. Burt et al, “Analysis of Wakefields in the ILC Crab Cavity”, EPAC 2006.

${ }^{6}$ MAFIA, CST GmbH, Darmstadt, [online] http://www.cst.com/.

${ }^{7}$ PLACET, CERN, Geneva, [online] https://savannah.cern.ch/projects/placet/.

${ }^{8}$ R. Jones et al, "Wakefields and Beam Dynamics Simulations for the $3.9 \mathrm{GHz}$ Cavities of the ILC”, LINAC 2006.

${ }^{9}$ R.Wanzenberg, TESLA 2001-33, September 2001.

${ }^{10}$ S. A. Heifets, S. A. Kheifets, "Coupling impedance in modern accelerators”, Rev. Mod. Phys. 63, 631 - 673 (1991)

${ }^{11}$ Microwave Studio, CST GmbH, Darmstadt, [online] http://www.cst.com/.

${ }^{12} \mathrm{G}$. Burt et al, "Effect of altering the cavity shape in infinitely periodic dipole cavities”, EUROTeV-Report-2007-003. 
${ }^{13}$ M. Liepe, "Pushing the Limits: RF Field Control at High Loaded Q”, PAC 2005.

${ }^{14}$ L. Xiao, Z. Li, K. Ko, SLAC-PUB-12409.

${ }^{15}$ C. Tenant et. al., "Experimental Investigation of Beam Breakup in the Jefferson Laboratory 10 kW FEL Upgrade Driver”, PAC 2005

${ }^{16}$ M. Church, FNAL ILC DocDB number 295, April 8, 2007.

${ }^{17}$ G. Burt, A. Dexter, P. Goudket, "Effect and Tolerances of RF Phase and Amplitude Errors in the ILC Crab Cavity”, EUROTeV-Report-2006-098

${ }^{18}$ V. Ayvazyan, S. Choroba et al, TESLA-FEL Report 2005-6. 